Revista Colombiana de Obstetricia y Ginecología Vol. 56 No.2 • 2005 • (141-146)

Artículo de Reflexión

\title{
HIPERTENSIÓN POSPARTO
}

\author{
Postpartum hypertension \\ Jesús Arnulfo Velásquez, M.D.* \\ Recibido: febrero 8/2005 - Revisado: mayo 16/2005 - Aceptado: junio 27/2005
}

\section{RESUIMEN}

Muy pocos estudios han investigado acerca de la fisiopatología y los cambios puerperales que favorecen la presentación de la hipertensión posparto. No existen datos que soporten la formulación de protocolos para el manejo de la hipertensión en este período, y la poca literatura disponible al respecto, se basa en experiencia no sistematizada.

Los trastornos hipertensivos del embarazo son un factor de riesgo importante para el desarrollo posterior de enfermedades cardiovasculares y de mortalidad asociada.

Estos trastornos se deben considerar como el inicio o tal vez la continuación de un sinnúmero de eventos subclínicos aún ignorados. Para la preeclampsia, el parto únicamente soluciona la "crisis" temporal que produce el embarazo, mas no alivia definitivamente el trastorno. Por lo tanto es necesario establecer estrategias adecuadas de vigilancia de la salud de la mujer.

Palabras clave: síndrome hipertensivo asociado al embarazo, hipertensión posparto.

\section{SUIMMARY}

There are very few studies investigating about the physiology and pathology of the different puerperal changes that can induce the onset and appearance of the postpartum hypertension.

Residente de tercer año, Departamento de Obstetricia y Ginecología, Facultad de Medicina, Universidad de Antioquia

Correo electrónico: susve@yahoo.com
Additionally there are not actual studies supporting the necessity for management protocols during this period, and the few available literature concerning these aspects has been based on nonorganized random experience.

The hypertensive conditions during pregnancy constitute a very important risk factor that may affect the odds for future development of cardiovascular disease and its related mortality.

These conditions must be considered like either the beginning or the consequence of an unlimited amount of subtle clinic events yet ignored.

In the preeclampsia cases the baby's delivery is only a temporary solution for the crisis that was triggered by pregnancy, but it is not the definitive cure for the condition. Henceforth, it is necessary to establish adequate strategies to closely evaluate and follow these women's health problems.

Key words: pregnancy induced hypertension, hypertensive syndrome related to pregnancy, post partum hypertension.

\section{INTRODUCCIÓN}

Los trastornos hipertensivos complican por lo menos $10 \%$ de los embarazos y aunque cada día más se conoce acerca de los eventos fisiopatológicos que rodean al síndrome hipertensivo asociado al embarazo, su causa no se ha identificado. ${ }^{1}$

En cuanto a la hipertensión posparto, no existe uniformidad en su manejo y seguimiento y son pocos los estudios que indagan acerca de su fisiopatología y los cambios puerperales que favorecen su presentación. 
No existen investigaciones que soporten la formulación de protocolos del manejo de la hipertensión en el posparto, y la poca literatura disponible al respecto se basa en experiencia no sistematizada.

A continuación se presentan algunas pautas y consideraciones sustentadas en aproximaciones fisiopatológicas y epidemiológicas, que deben motivar al personal de salud a investigar y a tener un criterio de responsabilidad en el seguimiento a largo plazo de la mujer que se presenta hipertensa en el posparto.

\section{EPIDEMIOLOGÍA}

Después de la violencia, las enfermedades cardiovasculares constituyen la principal causa de mortalidad en nuestro medio. ${ }^{2}$

Recientemente se han publicado estudios que confirman la relación de los trastornos hipertensivos de la gestación (hipertensión gestacional y preeclampsia) con el desarrollo posterior de enfermedad cardiovascular y de mortalidad asociada a ésta.

Wilson y colaboradores ${ }^{3}$ publicaron en el año 2003 un estudio de cohortes llevado a cabo en Noruega, con un seguimiento superior a 20 años de los grupos conformados: 1.197 controles, 1.197 hipertensas gestacionales y 1.199 preeclámpticas; reportaron un riesgo relativo de sufrir accidente cerebrovascular de 3,59 (IC95\% 1,04-12,4) en las mujeres que sufrieron preeclampsia. Además detectaron un riesgo aumentado de sufrir hipertensión arterial (HTA) posterior en ambos grupos, siendo ligeramente mayor en el grupo de preeclampsia, OR 1,21 (IC95\% 1,13-3,72).

Es de esperar que el mayor conocimiento de esta asociación derive en un diagnóstico más temprano y mejoría en el manejo, y tal vez así sea posible disminuir la morbilidad y la mortalidad asociadas.

En otro estudio noruego de seguimiento a 13 años, Irgens y colaboradores, ${ }^{4}$ reportaron que las pacientes que desarrollan preeclampsia por debajo de la semana 37 tienen un riesgo 8 veces superior de morir de enfermedad cardiovascular y 5 veces más de sufrir enfermedad coronaria, cuando se las compara con mujeres grávidas normotensas.

\section{FISIOPATOLOGÍA}

Se postula que el embarazo se debe considerar como una verdadera prueba de esfuerzo, durante la cual las demandas fisiológicas incrementadas actúan como "estrés" materno, que puede predecir la salud de una mujer más tarde en su vida. ${ }^{5}$

En términos generales, los síndromes gestacionales se desarrollan o aparecen cuando un órgano es incapaz de incrementar las demandas fisiológicas del embarazo, por este motivo el parto induce la remisión de éstos, aunque es transitorio. Más tarde, cuando los efectos del envejecimiento disminuyan las reservas limitadas de un órgano vulnerable, aparecerán trastornos clínicos como la hipertensión arterial y la diabetes.

El embarazo es un síndrome metabólico transitorio que predispone a disfunción endotelial. El síndrome metabólico es la confluencia de múltiples factores de riesgo de enfermedad cardiovascular, que se diagnostica con la presencia de más de 3 de los siguientes criterios según lo establece el Adult Treatment Panel (ATP III) (tabla 1).

\section{Tabla 1. Criterios diagnósticos del Adult Treatment Panel (ATP III) para síndrome metabólico. ${ }^{6}$}

* Hipertensión arterial (presión arterial $>130 / 85)$

* Obesidad abdominal (circunferencia abdominal $>88 \mathrm{~cm}$ en mujeres)

* Triglicéridos $>150 \mathrm{mg} / \mathrm{dl}$

* Lipoproteínas de alta densidad (HDL) $<50 \mathrm{mg} / \mathrm{dl}$

* Glicemia en ayunas $>110 \mathrm{mg} / \mathrm{dl}$

En el embarazo normal hay un gasto cardíaco aumentado, tendencia a la hipercoagulabilidad, aumento de marcadores de inflamación y, después de la semana 20 , resistencia a la insulina y dislipidemia. Todos estos factores son proaterogénicos y se encuentran más 
acentuados en la paciente que desarrolla hipertensión. Este síndrome metabólico persiste en el posparto y se evidencia como persistencia de un índice de masa corporal aumentado, aumento de PA y triglicéridos y disminución de las HDL. ${ }^{7}$

El síndrome metabólico se asocia al aumento de marcadores inflamatorios, reducción de la fibrinólisis, aumento de estrés oxidativo, presencia de microalbuminuria y anormalidades en la regulación autonómica. En este orden de ideas, se asocia con un aumento de 4 veces en el riesgo de sufrir enfermedad isquémica cardíaca, 2 veces en enfermedad cerebrovascular y de 5-9 veces de desarrollar diabetes mellitus. ${ }^{8}$

Después de un embarazo afectado por preeclampsia y antes que se haga evidente una enfermedad cardiovascular, hay evidencia de disfunción endotelial subclínica. Además, Satters y cols, encontraron que 15 a 25 años después de padecer preeclampsia se encuentran niveles aumentados de moléculas de adhesión vascular y evidencia de resistencia a la insulina. ${ }^{9}$

\section{Perfil hemodinámico de la puérpera con hipertensión}

Son muy pocos los estudios que indagan acerca del perfil hemodinámico de las puérperas hipertensas.

Walters reportó el comportamiento de la presión arterial en los primeros 5 días posparto de mujeres normales, observando que un aumento de ésta puede ser un hallazgo normal. ${ }^{10}$

MacGillivray y cols muestran que a las 6 semanas posparto la presión arterial fue más alta que en cualquier otro momento de la gestación; sin embargo, en este estudio no hay una observación longitudinal del puerperio. ${ }^{11}$

Al parecer existen dos factores importantes que ayudan a predecir la duración de la hipertensión arterial en el puerperio y son la severidad de la enfermedad antenatal y el inicio temprano de la preeclampsia. Es probable que la mayor perturbación hemodinámica explique la persistencia de la hipertensión arterial.

Ferrazzanni y cols, realizaron un estudio de cohortes para evaluar la duración de la hipertensión arterial en el puerperio de mujeres con trastornos hipertensivos; incluyeron 159 mujeres con diagnóstico de hipertensión gestacional y 110 pacientes con preeclampsia, el tiempo de normalización de la presión arterial en el primer grupo fue de 6 días y de 16 en el segundo. ${ }^{12}$

Por otro lado Walters, en un seguimiento de 62 pacientes con hipertensión durante el embarazo (30 con proteinuria), reportó una presión arterial de 150/100 al quinto día posparto. ${ }^{13}$ En la mayoría hubo una disminución de la presión arterial en los primeros días posparto seguido de un aumento posterior entre los días 3 y 6 , estableciendo como posible explicación de este fenómeno, el aumento del volumen intravascular secundario a la remoción de fluidos del espacio extravascular.

\section{CONSIDERACIONES CLIIINICAS}

La presencia de hipertensión en el puerperio obedece a una de las condiciones que figuran en la tabla 2.

\section{Tabla 2. Determinantes de la aparición} de hipertensión en el puerperio.

* Paciente que desarrolló preeclampsia o hipertensión gestacional y continúa hipertensa.

* Hipertensa crónica que desarrolló o no preeclampsia sobreagregada.

* Hipertensión que se presenta por primera vez en el puerperio.

Para realizar el diagnóstico de hipertensión arterial crónica después de un trastorno hipertensivo asociado al embarazo deben transcurrir 12 semanas. Es una obligación revaluar toda paciente con hipertensión en el puerperio. ${ }^{14}$

Idealmente se deben estudiar a todas las mujeres que se presentan hipertensión por primera en el puerperio y a aquéllas que desarrollan preeclampsia tempranamente, para así descartar causas secundarias de hipertensión como: trombofilia, enfermedad renal, aldosteronismo primario y feocromocitoma. 
En el posparto inmediato las pacientes deben continuar en control para detectar la presencia de síntomas premonitorios de eclampsia o encefalopatía hipertensiva, y para control de cifras tensionales, gasto urinario, y estado de hidratación.

El principal objetivo clínico del tratamiento es prevenir la hipertensión severa y sus secuelas y e desarrollo de eclampsia. Por lo menos 29\% de los casos de eclampsia ocurren en el posparto, la mayoría en las primeras 48 horas; $12 \%$ de los casos después de éstas, y sólo un 2\% después de 7 días. Los reportes suecos muestran que el 96\% ocurre en las primeras 20 horas. ${ }^{15}$

Teniendo en cuenta lo anterior y las consideraciones fisiopatológicas presentadas acerca de la duración de la hipertensión en el puerperio, resulta lógico sugerir que todas estas pacientes deberían ser dadas de alta por lo menos 4 días después del parto.

\section{CONSIDERACIONES DE MANEJO}

Aunque las recomendaciones de inicio de tratamiento farmacológico que se presentan en la literatura son arbitrarias, guardan coherencia con el limitado conocimiento disponible acerca de la fisiopatología. Por esto es urgente el diseño de investigaciones que permitan una aproximación a un manejo óptimo de la puérpera hipertensa.

A grosso modo son tres las prioridades que se deben atender:

a. Disminuir los factores relacionados con el síndrome metabólico.

b. Prevenir la eclampsia.

c. Prevenir y tratar la hipertensión severa y sus secuelas.

Para el tratamiento del síndrome metabólico se debe alentar a todas las maternas, especialmente a aquéllas que desarrollan hipertensión o diabetes gestacional, a modificar su estilo de vida; por este motivo se debe:

- Fomentar la actividad física.

- Controlar el peso.

- Instaurar una dieta rica en vegetales (antioxidantes), disminución de grasas, disminución de la ingesta de sal, eliminar el consumo de alcohol.
Con estas medidas lo que se busca es disminuir los factores nocivos para el endotelio y así tratar de retrasar la aparición de enfermedades debidas directamente a su disfunción. ${ }^{8}$

El sulfato de magnesio es la única droga que ha demostrado prevenir la eclampsia, y éste se debe instaurar en todos los casos de preeclampsia severa y mantenerse por lo menos 24 horas después del parto, o más si hay persistencia de síntomas premonitorios. ${ }^{15}$

La Sociedad Canadiense de Hipertensión recomienda iniciar tratamiento farmacológico de la hipertensión posparto en los siguientes casos. ${ }^{16}$

- Hipertensión severa.

- Presencia de síntomas: cefalea, visión borrosa.

- Presión arterial diastólica mayor de $100 \mathrm{mmHg}$, después de 3 días posparto y/o evidencia de daño de órgano.

Los fármacos propuestos son: metildopa, nifedipina y timolol. Sin embargo algunas experiencias británicas, ${ }^{17}$ aunque similares, hacen una aproximación un poco diferente. A continuación se presentan algunos parámetros en los que existe algún grado de acuerdo:

Iniciar tratamiento antihipertensivo si la presión arterial es $>150 / 100 \mathrm{mmHg}$ en los primeros 4 días posparto; lo anterior tiene dos justificaciones, ya que los mecanismos de autorregulación cerebral se pierden por encima de esta cifra de presión y ésta puede aumentar en los días posteriores.

Hablar de fármacos de primera línea es arbitrario, pues no hay estudios que traten específicamente este tópico y se desconoce en gran medida el perfil hemodinámico del puerperio; sin embargo se deben considerar como primera opción los siguientes grupos de medicamentos: beta bloqueadores, antagonistas de canales del calcio e inhibidores de la enzima convertidora de angiotensina (IECA). Algunos autores se inclinan por iniciar tratamiento con estos últimos.

La ventaja de los beta bloqueadores sobre la nifedipina estriba en que no tienen el efecto taquicardizante de ésta. La mayoría de fármacos usados son seguros en la lactancia, aunque hay reportes de efectos adversos neonatales con algunos beta 
bloqueadores que no se emplean en nuestro medio. Potencialmente todos pueden causar bloqueo beta adrenérgico en el neonato, sin embargo la Asociación Americana de Pediatría (APA) avala su uso. ${ }^{20}$

Aunque la APA no contraindica el uso de ninguno de los IECA, se debería preferir el enalapril, puesto que su excreción láctea es menor que la de captopril.

La paciente que es hipertensa crónica debe volver a su esquema prenatal, teniendo en cuenta que puede requerir un aumento en su dosificación, por los cambios señalados. ${ }^{21}$

Las pacientes hipertensas en el puerperio se deben dar de alta después del tercer o cuarto día posparto, ya que el riesgo de hemorragia intracraneana y de eclampsia es bajo después del tercer día. Se debe dar alta a la paciente siempre y cuando tenga una presión arterial $<150 / 100$.

Todas las pacientes deben ser evaluadas como mínimo cada siete días durante las primeras doce semanas posparto, para disminuir o aumentar la dosificación farmacológica, entregando siempre instrucciones claras sobre sintomatología de encefalopatía hipertensiva y de eclampsia en la primera semana posparto.

Si después de 12 semanas de seguimiento la paciente persiste hipertensa o aún requiere de medicamentos antihipertensivos, se debe catalogar como hipertensa crónica y debe ingresar a un programa de hipertensión arterial.

La paciente que logra resolver su hipertensión en las 12 semanas siguientes al parto, que son la mayoría, se deben instruir sobre control de factores de riesgo cardiovascular, y por lo menos una vez al año se les debería realizar tamizaje para hipertensión arterial, dislipidemia y diabetes. Sin embargo se debe aclarar que no hay estudios que señalen cada cuánto se debe hacer tamizaje en una paciente que padeció algún trastorno hipertensivo durante la gestación. En la tabla 3 se presentan los medicamentos más usados en la hipertensión posparto.

\section{CONCLUSIONES}

Los trastornos hipertensivos del embarazo son un factor de riesgo importante para el desarrollo poste-

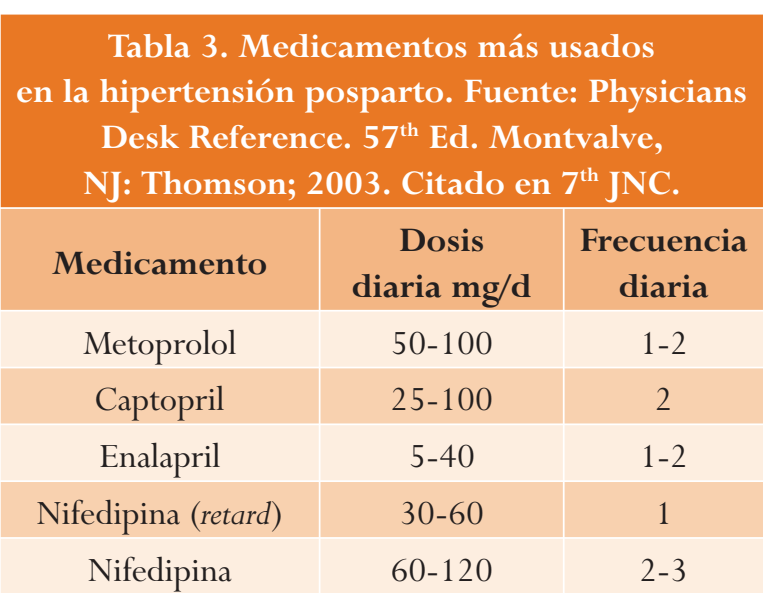

rior de enfermedades cardiovasculares y de mortalidad asociada. Además, el embarazo por sí mismo se convierte en un reto fisiológico fundamental y determinante de la salud femenina.

Deben entenderse los trastornos hipertensivos de la gestación como el inicio o tal vez la continuación de un sinnúmero de eventos subclínicos que aún se ignoran. El parto únicamente soluciona la "crisis" temporal que produce el embarazo mas no alivia definitivamente el trastorno. Por tanto es necesario estar atentos y establecer estrategias adecuadas de vigilancia de la salud de la mujer.

\section{REFERENCIAS}

1. Report of the National High Blood Pressure Education Program Working Group on High Blood Pressure in Pregnancy. Am J Obstet Gynecol 2000;183:S1-S22.

2. Departamento Administrativo Nacional de Estadísticas, 2002.

3. Wilson BJ, Watson MS, Prescott GJ, Sunderland S, Campbell DM, Hannaford P, et. al. Hypertensive diseases of pregnancy and risk of hypertension and stroke in later life: results from cohort study. BMJ 2003;326:845.

4. Irgens HU, Reisaeter L, Irgens LM, Lie RT. Long term mortality of mothers and fathers after preeclampsia: population based cohort study. BMJ 2001;323:1213-7.

5. Williams D. Pregnancy: a stress test for life. Curr Opin Obstet Gynecol 2003;15:465-71.

6. National Cholesterol Education Program (NCEP), Expert panel on detection, evaluation, and treatment 
of high blood cholesterol in adults (Adult treatment panel III). Third report of the National Cholesterol Education Program (NCEP) Expert Panel on Detection, Evaluation, and Treatment of High Blood Cholesterol in Adults (Adult treatment panel III) final report. Circulation 2002;106:3143-421.

7. Pouta A, Hartikainen AL, Sovio U, Gissler M, Laitinen J, McCarthy MI, et al. Manifestations of metabolic syndrome after hypertensive pregnancy. Hypertension 2004;43:825-31.

8. Chobanian AV, Bakris GL, Black HR, Cushman WC, Green LA, Izzo JL Jr, et al. Seventh report of the Joint National Committee on prevention, detection, evaluation and treatment of high blood pressure. Hypertension 2003;1206-52.

9. Chambers JC, Fusi L, Malik IS, Haskard DO, De Swiet M, Kooner JS. Association of maternal endothelial dysfunction with preeclampsia. JAMA 2001;285:1607-12.

10. Walters BN, Thompson ME, Lee A, de Swiet M. Blood pressure in the puerperium. Clin Sci 1986;71:589-94.

11. MacGillivray I, Rose GA, Rowe B. Blood pressure survey in pregnancy. Clin Sci 1969;37:395-407.
12. Ferrazzani S, De Carolis S, Pomini F, Testa AC, Mastromarino C, Caruso A. The duration of hypertension in the puerperium of preeclamptic women: relationship with renal impairment and web of delivery. Am J Obstet Gynecol 1994;17:506-12.

13. Walters BN, Walters T. Hypertension in the puerperium. Lancet 1987;2: 330.

14. Douglas KA, Redman CW. Eclampsia in the United Kingdom. BMJ 1994; 309:1395-400.

15. Altman D, Carroli G, Duley L, Farrell B, Moodley J, Neilson J, et al. Do women with pre-eclampsia, and their babies, benefit from magnesium sulphate? The magpie trial: a randomized placebo-controlled trial. Lancet 2002;359:1877-90.

16. Rey E, LeLorier J, Burgess E, Lange IR, Leduc L. Report of the canadian hypertension society consensus conference: 3. Pharmacologic treatment of hypertensive disorders in pregnancy. CMAJ 1997;157:1245-54.

17. Tan LK, Swiet M. The management of postpartum hypertension. BJOG 2002;109:733-6.

18. Briggs GG. Drug effects on the fetus and breast fed infant. Clin Obstet Gynecol 2002;45:6-21. 